\section{Case Reports in Ophthalmology}

Case Rep Ophthalmol 2020;11:612-619

This article is licensed under the Creative Commons Attribution-NonCommercial 4.0 International License (CC BY-NC) (http://www.karger.com/Services/OpenAccessLicense). Usage and distribution for commercial purposes requires written permission.

\title{
Multimodal Imaging of Large Optic Disc Coloboma: A Report of Three Cases
}

\author{
Sophia El Hamichic ${ }^{\mathrm{a}, \mathrm{b}}$ Dhariana Acón ${ }^{\mathrm{b}}$ Timothy G. Murray ${ }^{\mathrm{a}}$ \\ Audina M. Berrocal ${ }^{b}$ \\ aMurray Ocular Oncology and Retina, Miami, FL, USA; bepartment of \\ Ophthalmology, Bascom Palmer Eye Institute, University of Miami Miller School \\ of Medicine, Miami, FL, USA
}

\section{Keywords}

Optical coherence tomography angiography · Coloboma · Optic disc coloboma · Optic nerve coloboma

\section{Abstract}

Optic disc coloboma (ODC) is a rare congenital anomaly of the optic nerve. Optical coherence tomography (OCT) helps to monitor the complications when there is associated retinoschisis, retinal detachment, or peripapillary neovascularization, while being minimally invasive. OCT angiography could help to better understand this entity from a vascular perspective. We report multimodal imaging of 3 children with large ODC associated with cat eye syndrome, CHARGE syndrome (coloboma, heart defects, atresia choanae, growth retardation, genital abnormalities, and ear abnormalities), and branchio-oculo-facial syndrome.

(C) 2020 The Author(s)

Published by S. Karger AG, Basel

\section{Introduction}

Optic disc coloboma (ODC) is a rare congenital defect resulting from incomplete proximal optic fissure closure $[1,2]$. It may occur as an isolated finding or associated with other eye 


\section{Case Reports in Ophthalmology}

\begin{tabular}{l|l} 
Case Rep Ophthalmol 2020;11:612-619 \\
\hline DOI: 10.1159/000509848 & $\begin{array}{l}\text { @) 2020 The Author(s). Published by S. Karger AG, Basel } \\
\text { www.karger.com/cop }\end{array}$ \\
\hline
\end{tabular}

El Hamichi et al.: Optical Coherence Tomography Angiography in Large Colobomas

colobomas, including coloboma of the lens, iris coloboma, and retinochoroidal coloboma [3]. Visual acuity is affected differently depending on the ODC itself or secondary complications.

Optical coherence tomography (OCT) is a helpful tool to monitor these patients when there is an association of retinoschisis and retinal detachment. OCT angiography (OCTA) is helpful in differentiating between ODC from other optic nerve defects including morning glory syndrome [4]. OCTA has not been thoroughly explored regarding vascular physiology of colobomas. It will also be beneficial to know whether OCTA helps to establish a better understanding of the pathophysiology of the coloboma's vascularization system and the possibility of any correlation with visual function, as retinal and choroidal vasculature may impact vision.

Only a few studies describing the multimodal imaging aspects of congenital optic disc anomalies have been published.

\section{Subjects and Methods}

A retrospective study of patients who presented to the Department of Pediatric Retina at Bascom Palmer Eye Institute, Miami, Florida between January 2019 and January 2020 was performed. Six eyes of 3 patients were identified. All patients were seen in the clinic and brought to the operating room for examination under general anesthesia (EUA) to perform a full ophthalmological examination, fundus photography, fluorescein angiography (FA), OCT, and OCTA using the Heidelberg Spectralis Flex Module. Then, necessary treatment was implemented as needed. The OCT and OCTA were performed by trained experienced photographers. The visual acuity in this paper is provided in the case descriptions whenever measurement in these children was possible.

\section{Results}

\section{Case 1}

A 3-year-old female with a history of plagiocephaly and tetralogy of Fallot who had undergone heart surgery was referred by the pediatric ophthalmologist for cat eye syndrome OU. She had partial tetrasomy (four copies) of 22q11.1q11.21 chromosome by microarray.

During EUA, intraocular pressure (IOP) was $12 \mathrm{~mm} \mathrm{Hg} \mathrm{OU} \mathrm{and} \mathrm{axial} \mathrm{length} \mathrm{was} 20.1 \mathrm{~mm}$ in both eyes. Anterior segment evaluation showed inferior coloboma of the iris in both eyes. Indirect ophthalmoscopy of the posterior segment of the eye demonstrated tilted optic disc and bilateral coloboma of nerve, retina, and choroid bilaterally; in the right eye exudation was noticed starting superotemporally and compromising the macula with an adjacent hemorrhage consistent with choroidal neovascularization (CNV) (OD depicted in Fig. 1a). FA revealed staining of the coloboma OU, with blockage from the hemorrhage, and leakage from the neovascular membrane in the right eye (OD depicted in Fig. 1b). Three intravitreal bevacizumab injections and a subsequent subtenon triamcinolone injection were administered to treat the patient's right eye. Additionally, laser photocoagulation treatment was needed due to persistent CNV. One year later the CNV had resolved with a best corrected visual acuity of 20/150. During the latest EUA, OCT showed retinochoroidal scleral excavation in the area of the ODC, with a fine layer of retina representing the intercalary membrane directly covering the sclera OU (OD depicted in Fig. 1c). In the right eye, CNV with cystic edema temporally to the ODC was noticed (Fig. 1e). OCTA could not capture clear pictures of the ODC due to its depth (Fig. 1f); however, we noticed a neovascular network growing from the choroid 


\section{Case Reports in Ophthalmology}

Case Rep Ophthalmol 2020;11:612-619

DOI: 10.1159/000509848

(c) 2020 The Author(s). Published by S. Karger AG, Basel www.karger.com/cop

El Hamichi et al.: Optical Coherence Tomography Angiography in Large Colobomas

vasculature through Bruch's membrane corresponding to the CNV at the temporal edge of the ODC in the right eye (Fig. 1d). This OCTA finding is difficult to differentiate from a projection artifact and segmentation errors. The patient continues to be monitored closely.

\section{Case 2}

A 14-year-old female with a history of CHARGE syndrome (coloboma, heart defects, atresia choanae, growth retardation, genital abnormalities, and ear abnormalities) was referred for retinal evaluation due to presence of retinal detachment in the right eye. Her best corrected visual acuity was 20/400 OD and 20/60 OS. During the EUA, IOP was $12 \mathrm{~mm} \mathrm{Hg}$ OD

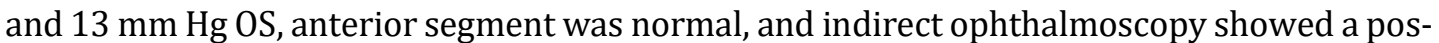
terior pole coloboma of the right eye with a superior focal retinal detachment. In the left eye a chorioretinal coloboma with an attached retina was found (Fig. 2a). FA demonstrated bilateral staining of the coloboma with peripheral nonperfusion (OS depicted in Fig. 2b). A pars plana vitrectomy was performed OD with silicone oil tamponade. Eleven months after surgery the patient developed a cataract. At this time, OCT was performed in the left eye and showed retinochoroidal scleral excavation in the area of the ODC, with the retina reduced to a fine intercalary membrane covering the sclera (Fig. 2c), while in OCTA a decrease in capillary perfusion was suspected OS (Fig. 2d); however, it is difficult to differentiate from a signal void. The OCTA images were not completely conclusive as the quality was poor due to the significant depth of the coloboma. Phacoemulsification was then performed with silicone oil removal. During the surgery, retinal holes with subretinal fluid were noticed superiorly, laser photocoagulation was applied along with $\mathrm{C}_{3} \mathrm{~F}_{8} 18 \%$ gas tamponade ensuring retinal reattachment, then intravitreal bevacizumab injection was given. At the most recent follow-up, the retina remained attached, with a visual acuity of 20/200 OD and 20/60 OS.

\section{Case 3}

A 3-year-old female with a history of branchio-oculo-facial syndrome underwent EUA.

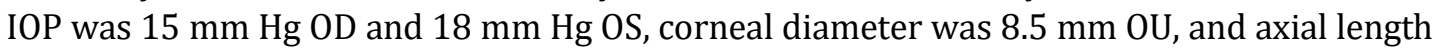
was $16.5 \mathrm{~mm}$ OD and $19.5 \mathrm{~mm}$ OS. The anterior segment showed keyhole iris with inferior coloboma, prominent vascularization, and mild pupillary membrane $\mathrm{OD}$, and coloboma of the iris OS, with cataract OU. Indirect ophthalmoscopy OD demonstrated presence of persistent fetal vasculature, coloboma of the optic nerve, diffuse retinal pigment epithelium hypertrophy in the macula, and tortuous vessels with subretinal fluid in the four quadrants. Indirect ophthalmoscopy OS revealed coloboma of the optic nerve associated with inferior chorioretinal coloboma (OS depicted in Fig. 3a). Two years after initial evaluation, evolution in the left eye was unfavorable, with no view to the posterior segment of the eye. Axial length was $16.0 \mathrm{~mm}$ OD and $20.4 \mathrm{~mm}$ OS. We noticed the presence of subretinal fluid OS, and FA showed patchy perfusion with speckled fluorescence (Fig. $3 \mathrm{~b}$ ). The patient received multiple laser photocoagulation treatments, intravitreal injections of bevacizumab, and subtenon triamcinolone injections OS, on a treat-and-adjust interval. OCT OS was performed during the most recent followup and showed the coloboma with the intercalary membrane covering the sclera (Fig. 3c) and subretinal fluid superior to the ODC (Fig. 3d). OCTA was also performed OS, but the images were difficult to interpret overall; due to the significant depth of the coloboma, we could only see the edge of the ODC in some portions showing decreased capillary perfusion, however this is difficult to differentiated from artifact (Fig. 3e, f). The patient continues to be monitored closely. 


\section{Case Reports in Ophthalmology}

\section{Discussion and Conclusions}

ODC is a rare congenital disorder resulting from incomplete closure of the embryonic fissure during the 5 th to 7 th week of gestational age $[1,2]$. The prevalence of ODC in the general population is about 0.14 , with $50 \%$ of patients having bilateral disease [5]. The closure defect is often filled with glial tissue. ODC may be an isolated defect or associated with iris coloboma, lens coloboma, or retinochoroidal coloboma [3]. Visual acuity varies according to the degree of foveal involvement and overlying retinoschisis or detachment that can be associated with this condition [6].

ODC can be sporadic or inherited in an autosomal dominant pattern, with reported associations with variants in PAX2 [6]. It can also be associated with different syndromes: cat eye syndrome (as seen in case 1), CHARGE syndrome (depicted in case 2), branchio-oculo-facial syndrome (example seen in case 3), first arch syndrome that includes Treacher Collins syndrome and Patau syndrome, Fraser syndrome, amniotic band syndrome, Aicardi syndrome, and others $[4,7]$.

There are only a few studies describing the OCT aspects of ODC. Spectral-domain OCT demonstrates sclera just beneath the retinal tissue. When retinoschisis is present, it is found above the excavation. Spectral-domain OCT also shows that the retinochoroidal-scleral excavation in the area of the ODC is relatively shallow compared to that observed in morning glory syndrome and peripapillary staphyloma. Furthermore, according to these studies, there is a considerable overlap between the OCT findings in ODC and optic disc pits $[6,8,9]$.

OCTA allows the evaluation of peripapillary vascular network in different layers. In normal eyes, there is a dense microvascular network with no focal capillary dropout [10]. One study reported that OCTA in ODC shows absence of a radial peripapillary microvascular network and a region of capillary dropout. This finding was similar in optic disc pits and in contrast to morning glory syndrome, where the microvascular peripapillary network is dense $[9$, $11]$.

Other studies demonstrated that there is a significant decrease in capillary perfusion density at the inside disc, inferior nasal, and temporal regions in optic disc pits eyes. Furthermore, these studies showed that in morning glory syndrome, besides the increased density of the radial peripapillary capillary network, there is an absence of superficial capillary plexus and a rarefaction of the vasculature of the peripapillary area adjacent to the disc anomaly at the deep capillary plexus and at the level of choriocapillaris $[12,13]$.

OCTA as a novel ancillary test is promising as it enables the analysis of the vascular network of different layers of the eye. However, in the studies discussed above, OCTA only described small ODC and optic nerve pit. In fact, the currently available OCTA is not capable of capturing large ODC and the different vascular layers cannot be analyzed, which is the case in our patients; we have included the best captures obtained in Figures 1-3. In large ODCs, OCT is helpful to monitor the retinoschisis and the CNV that could accompany this condition; in contrast, OCTA is of limited interest.

\section{Acknowledgment}

The authors thank Giselle De Oliveira, photographer, for providing this paper with images. 


\section{Case Reports in Ophthalmology}

\section{Statement of Ethics}

This case series was conducted in accordance with the Declaration of Helsinki. The collection and evaluation of all protected patient health information was performed in a manner compliant with the Health Insurance Portability and Accountability Act. Written informed consent was obtained from the patients' parents/legal guardians prior to performing the procedure, including permission for publication of the images included herein.

\section{Conflict of Interest Statement}

The authors have no conflicts of interest to declare.

\section{Funding Sources}

None.

\section{Author Contributions}

S. El Hamichi: data acquisition, data analysis, data interpretation, drafting of the work. D. Acón: data analysis, data interpretation, drafting of the work. T.G. Murray: design of the work, revising the work critically for important intellectual content. A.M. Berrocal: conception and design of the work, revising the work critically for important intellectual content.

\section{References}

1 Pagon RA. Ocular coloboma. Surv Ophthalmol. 1981 Jan-Feb;25(4):223-36.

2 Pedler C. Unusual coloboma of the optic nerve entrance. Br J Ophthalmol. 1961 Dec;45(12):803-7.

3 Babalola YO, Olawoye 00, Idam PO. Optic disc coloboma in two Nigerian siblings: case report and review of literature. Niger J Clin Pract. 2017 Nov;20(11):1505-9.

4 Vegunta S, Patel BC. Optic Nerve Coloboma. In: StatPearls [Internet]. Treasure Island: StatPearls Publishing; 2020.

5 Amador-Patarroyo MJ, Pérez-Rueda MA, Tellez CH. Congenital anomalies of the optic nerve. Saudi J Ophthalmol. 2015 Jan-Mar;29(1):32-8.

6 Jeng-Miller KW, Cestari DM, Gaier ED. Congenital anomalies of the optic disc: insights from optical coherence tomography imaging. Curr Opin Ophthalmol. 2017 Nov;28(6):579-86.

7 Dumitrescu AV, Milunsky JM, Longmuir SQ, Drack AV. A family with branchio-oculo-facial syndrome with primarily ocular involvement associated with mutation of the TFAP2A gene. Ophthalmic Genet. 2012 Jun; 33(2):100-6.

8 Lee KM, Woo SJ, Hwang JM. Evaluation of congenital excavated optic disc anomalies with spectral-domain and swept-source optical coherence tomography. Graefes Arch Clin Exp Ophthalmol. 2014 Nov;252(11): 1853-60.

9 Cennamo G, Rossi C, Ruggiero P, de Crecchio G, Cennamo G. Study of the radial peripapillary capillary network in congenital optic disc anomalies with optical coherence tomography angiography. Am J Ophthalmol. 2017 Apr;176:1-8.

10 Akil H, Falavarjani KG, Sadda SR, Sadun AA. Optical coherence tomography angiography of the optic disc; an Overview. J Ophthalmic Vis Res. 2017 Jan-Mar;12(1):98-105.

11 Cennamo G, Montorio D, Breve MA, Morra VB, Cennamo G. Optical coherence tomography angiography in contractile morning glory syndrome. Eur J Ophthalmol. 2019 doi: 10.1177/1120672119864010 [Epub ahead of print]. 


\section{Case Reports in Ophthalmology}

\begin{tabular}{l|l}
\hline DOI: 10.1159/000509848 & $\begin{array}{l}\text { (c) 2020 The Author(s). Published by S. Karger AG, Basel } \\
\text { www.karger.com/cop }\end{array}$ \\
\hline
\end{tabular}

El Hamichi et al.: Optical Coherence Tomography Angiography in Large Colobomas

12 Jiang S, Turco B, Choudhry N. Vascular perfusion density mapping using optical coherence tomography angiography comparing normal and optic disk pit eyes. Retin Cases Brief Rep. 2019 doi: 10.1097/ ICB.0000000000000908 [Epub ahead of print].

13 Romano F, Giuffrè C, Arrigo A, Corbelli E, Battaglia Parodi M, Bandello F. Case Report: Optical Coherence Tomography Angiography in Morning Glory Disc Anomaly. Optom Vis Sci. 2018 Jun;95(6):550-2.
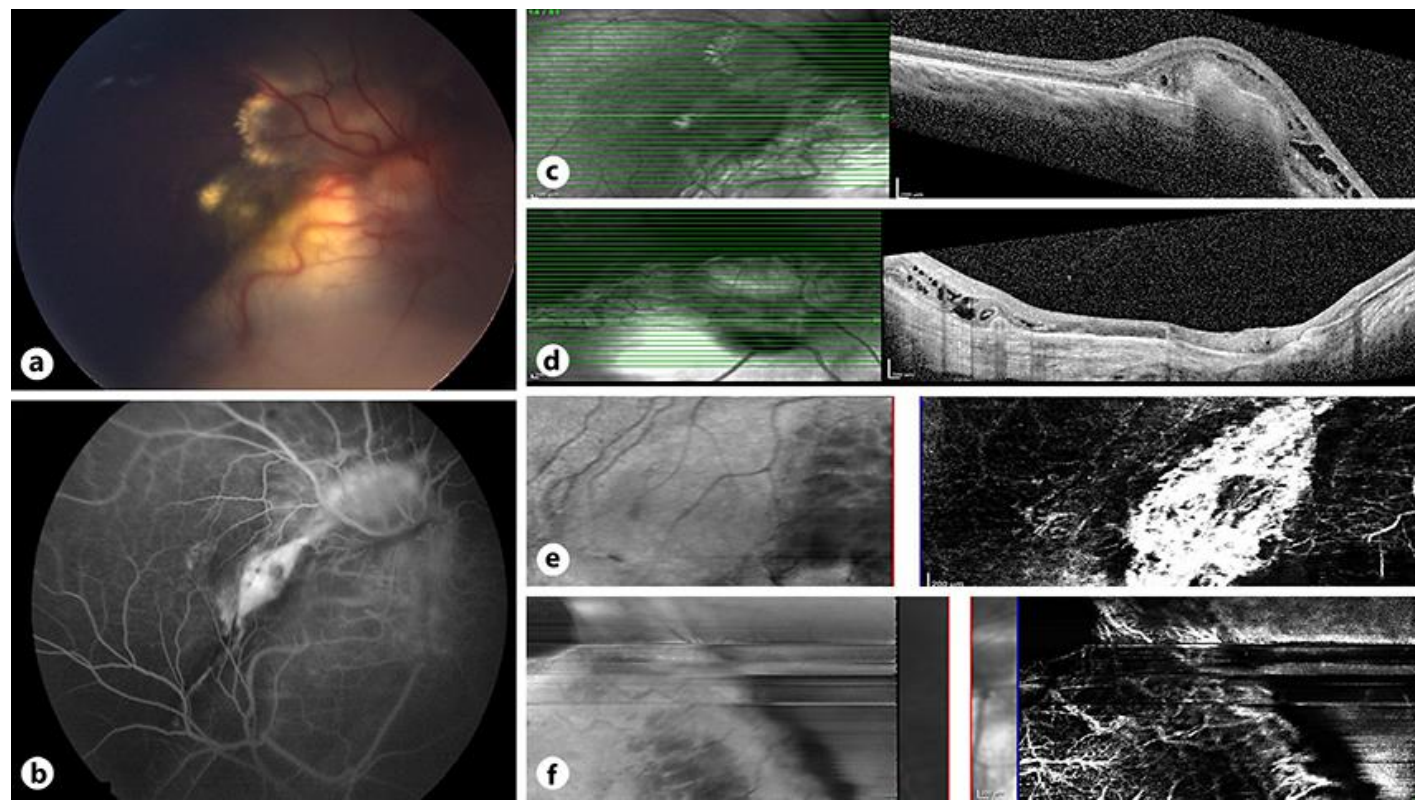

Fig. 1. Case 1. a Color fundus photograph of the right eye showing the ODC; note the CNV at the superotemporal edge of the coloboma. $\mathbf{b}$ FA of the right eye showing staining of the coloboma and late leakage of the CNV. c OCT of the CNV superotemporal to the coloboma showing a hyperreflective nodular lesion above the retinal pigment epithelium with intraretinal edema. $\mathbf{d}$ OCT of the coloboma showing retina directly on top of the sclera with cystoid edema temporally. e OCTA (avascular complex) of the CNV membrane. f OCTA (avascular complex) of the coloboma not showing clear details of the vasculature. CNV, choroidal neovascularization; FA, fluorescein angiography; OCT, optical coherence tomography; OCTA, optical coherence tomography angiography; ODC, optic disc coloboma. 
Case Reports in Ophthalmology
Case Rep Ophthalmol 2020;11:612-619

DOI: $10.1159 / 000509848$

(c) 2020 The Author(s). Published by S. Karger AG, Basel www.karger.com/cop

El Hamichi et al:: Optical Coherence Tomography Angiography in Large Colobomas
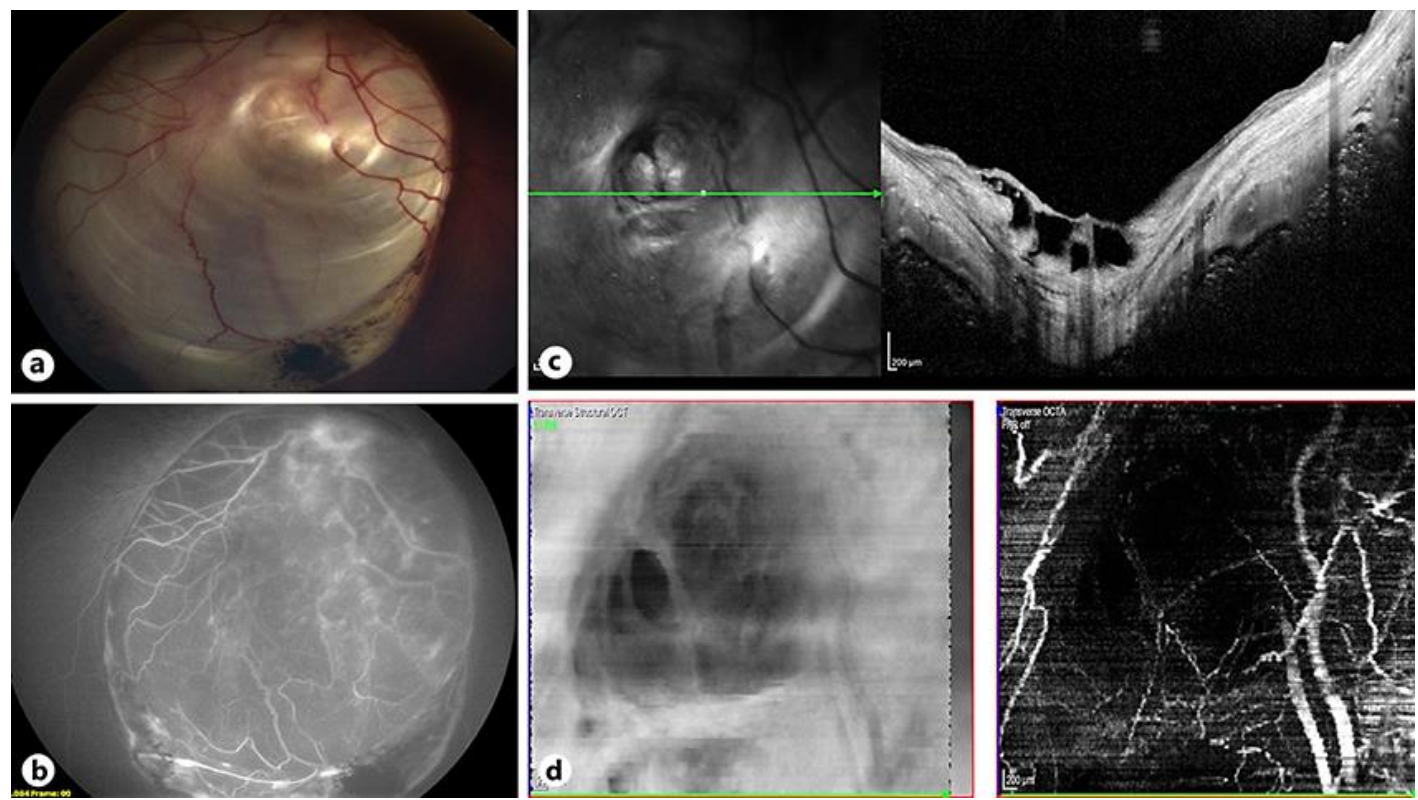

Fig. 2. Case 2. a Color fundus photograph of the left eye showing the ODC. b FA of the left eye showing late staining of the coloboma. c OCT of the coloboma showing a fine layer of retina directly on top of the sclera with cystoid edema. d OCTA (RET) of the coloboma not showing clear details, but a decrease in vasculature can be suspected. FA, fluorescein angiography; OCT, optical coherence tomography; OCTA, optical coherence tomography angiography; ODC, optic disc coloboma. 


\section{Case Reports in Ophthalmology}
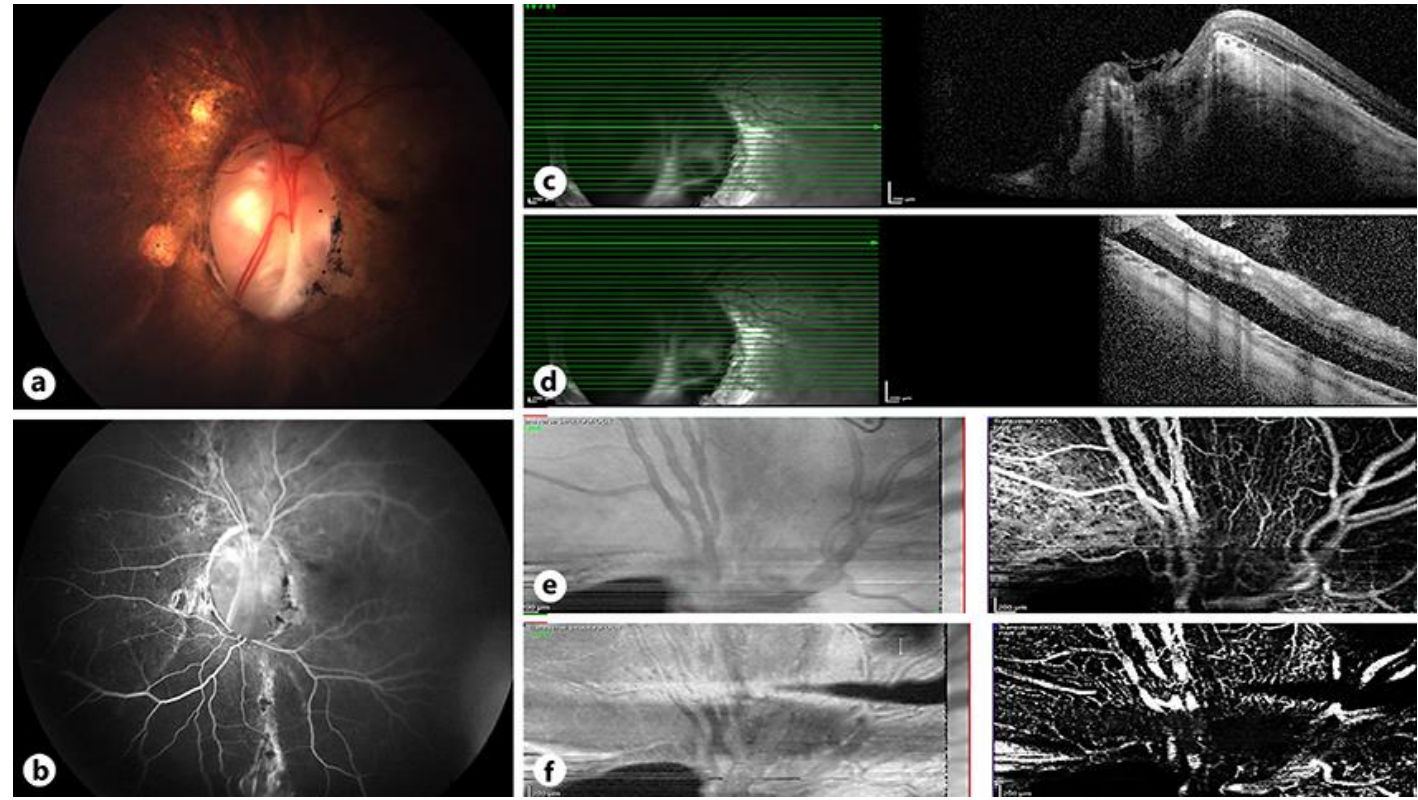

Fig. 3. Case 3. a Color fundus photograph of the left eye showing the ODC. b FA of the left eye showing staining of the coloboma and a vertical inferior hypofluorescent line corresponding to a chorioretinal coloboma. c OCT of the coloboma showing a fine layer of retina on top of the sclera. $\mathbf{d}$ OCT superior to the coloboma demonstrating subretinal fluid. e, f OCTA of the coloboma (RET and deep vascular complex, respectively) showing decreased vasculature although the details cannot be fully appreciated. FA, fluorescein angiography; OCT, optical coherence tomography; OCTA, optical coherence tomography angiography; ODC, optic disc coloboma. 